Recherches en didactique des langues et des cultures

Les cahiers de l'Acedle

2 | 2006

Recherches en didactique des langues. Actes du colloque de Lyon

\title{
Spécificités des techniques d'enseignement par rapport aux publics, mythe ou réalité ?
}

\section{Anne Chateau}

\section{CpenEdition}

1 Journals

Édition électronique

URL : http://journals.openedition.org/rdlc/5131

DOI : $10.4000 /$ rdlc. 5131

ISSN : 1958-5772

Éditeur

ACEDLE

Référence électronique

Anne Chateau, «Spécificités des techniques d'enseignement par rapport aux publics, mythe ou réalité ? ", Recherches en didactique des langues et des cultures [En ligne], 2 | 2006, mis en ligne le 06 décembre 2006, consulté le 11 septembre 2019. URL : http://journals.openedition.org/rdlc/5131 DOI : $10.4000 /$ rdlc.5131

Ce document a été généré automatiquement le 11 septembre 2019.

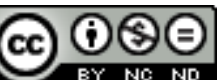

Recherches en didactique des langues et des cultures is licensed under a Creative Commons AttributionNonCommercial-NoDerivatives 4.0 International License 


\title{
Spécificités des techniques d'enseignement par rapport aux publics, mythe ou réalité ?
}

\author{
Anne Chateau
}

\section{Introduction}

1 Le Contexte théorique dans lequel s'inscrit cet article est celui de la didactique praxéologique qui, permet au didacticien d'observer: "des problèmes qui se posent sur le terrain et [d'aller] chercher dans les théories des sciences de fondement des réponses à ces problèmes qu'il mettra à l'épreuve de la réalité du terrain." (M.-F. Narcy-Combes, $2005: 17)$. Cet article va en effet s'appuyer essentiellement sur une recherche menée à l'université Henri Poincaré (UHP) à Nancy sur une période de 3 ans (1998-2001) auprès d'un public du secteur Lansad ${ }^{1}$ (doctorants scientifiques représentatifs de plusieurs spécialités). Ce projet, visant à répondre aux besoins en communication orale scientifique en anglais des jeunes chercheurs (cf. Chateau, 2003), a mis en lumière l'importance de l'adaptation des formations proposées aux spécificités des publics.

2 Inversement, la possibilité de transposer d'un contexte à l'autre certains des paramètres ou outils conçus dans le cadre du dispositif proposé à l'UHP a également été révélée au cours de cette recherche-action, puisque le dispositif, bien que prévu au départ pour une population donnée d'étudiants, a ensuite pu être étendu à un autre public (au sein de la même université) et servir de modèle pour un troisième type de public (dans le cadre d'un organisme externe de formation); certains des éléments pédagogiques creés ont été utilisés, après une adaptation, auprès d'étudiants d'une autre université.

3 L'adaptation nécessaire aux spécificités des publics n'est donc peut-être que superficielle (au sens premier du terme) et limitée à quelques éléments que cet article essaiera de définir. En outre, il serait peut-être intéressant de comprendre ce qui, dans les techniques utilisées auprès de ces publics, pourrait être repris et adapté à d'autres. 


\section{Contexte environnemental}

4 L'UHP est une université scientifique comprenant des Facultés de Médecine, Odontologie, Pharmacie, Sciences et Faculté du Sport, trois IUT, ainsi que des Écoles d'ingénieurs. Les publics (environ 17500 étudiants) auxquels les enseignants de langues y sont confrontés sont donc très divers et de niveaux variés (niveaux $\mathrm{Bac}+1$ à $\mathrm{Bac}+8$ ).

5 Dans cette université, un centre de ressources documentaires à l'usage des enseignants de langues de l'Université, le Crelens (Centre de Ressources pour l'Enseignement des Langues Étrangères aux Non-Spécialistes) a pour mission de proposer des réponses aux besoins pédagogiques et de recherche exprimés par les composantes de l'UHP, et d'impulser des actions pouvant inclure des enseignants issus de toutes les composantes et ayant des répercussions sur les différents publics. Ce centre a donc un rôle de fédérateur d'actions en réseau, ce qui a eu un impact non négligeable sur la façon dont la recherche a été menée (voir Magnet, 1999: 90; Chateau, 2003: 67; ou encore J.-P. Narcy-Combes, 2005 : 92, pour plus de détails sur la notion de réseau). Neuf enseignants, intervenant dans sept composantes différentes - à la fois géographiquement, mais, également du point de vue des spécialités de leurs étudiants - participaient en effet au projet la première année, même si l'équipe a pour diverses raisons été un peu réduite par la suite. ${ }^{2}$

6 En outre, même si "le bien-fondé d'allier apprentissage de la langue et contenu spécialisé a été largement discuté" (Péchou, 2005: 100), dans le cas décrit ici cette nécessité était absolument impérative. En 1998, en effet, les instances de l'UHP ont fait part de leur volonté institutionnelle d'améliorer les compétences de communication scientifique orale en anglais des étudiants chercheurs. Cette volonté nécessitait de trouver une réponse durable et imposait, de la part de l'équipe souhaitant s'investir dans le projet, une solide connaissance des habitudes de communication de la communauté scientifique.

\section{Contexte culturel}

7 Le public cible de cette recherche était composé de jeunes chercheurs scientifiques; comme plusieurs chercheurs du secteur Lansad l'ont indiqué, en général les scientifiques français ont un très mauvais niveau de communication (voir Ginet, 1994 ; Guyon, 1996 ; Ogrizek et al., 1996; ou Rowley-Jolivet, 1997 par exemple). Il était donc nécessaire, pour parvenir à répondre aux besoins spécifiques de ces étudiants, de bien connaître le contexte dans lequel ils allaient être amenés à communiquer, à la fois du point de vue de la langue, mais également sur le plan culturel. Le terme 'culture' est ici utilisé "dans son acception anthropologique et expérientielle." (Isani, 2004:6). Ainsi que le rappelle cet auteur, la compétence culturelle est en effet l'une des composantes de la compétence de communication. Ceci est à rapprocher de ce que déclare Rowley-Jolivet: "les normes moins exigeantes de la maîtrise de la langue anglaise qui prévalent dans la communication scientifique orale [...] tendent à indiquer que la compétence primordiale requise en mode oral est culturelle ou rhétorique plutôt que simplement linguistique" (1997: 35).

8 Les apprenants auxquels nous allions nous adresser relevant d'un domaine bien spécifique et ayant à utiliser ce qui dans le domaine anglo-saxon est qualifié de English for Specific purposes (anglais à objectifs spécifiques ou langue appliquée), il nous apparaissait 
donc impératif de bien connaître le genre de la communication scientifique orale. Pour trouver une solution adaptée aux besoins de ces apprenants, et apporter le nécessaire cadrage théorique à cette demande issue du terrain, la recherche-action nous semblait la meilleure façon d'éviter des solutions pédagogiques inadaptées et s'apparentant plus à des 'recettes', inadaptées aux besoins d'un tel public.

\section{Une recherche-action}

La recherche-action - "recherche liée à la réalisation pratique de résolutions de problèmes de terrain où la réflexion a une dimension aussi bien pratique que critique" (Narcy, 1998 : 230) paraissait le mode de fonctionnement le plus approprié pour résoudre le problème institutionnel qui nous était posé. Ainsi que nous l'avons montré par ailleurs (Château \& Georges, 2002) le projet mené à l'UHP comportait de nombreux paramètres. La problématique à construire était donc complexe, et une confrontation permanente entre théorie et pratique - avec la mise en place d'expérimentations suivie de retours et de modifications éventuelles - semblait être un moyen adéquat d'y parvenir.

Le cadre théorique que nous avons dû bâtir pour mener à bien cette recherche-action était composé de paramètres divers. Il tenait compte d'abord du public, et donc des facteurs pouvant influer sur son apprentissage (motivation, individualisation, fonctionnement cognitif). Cet aspect de la recherche relève de la didactique des langues. Nous avons également dû tenir compte de la communication scientifique orale en anglais, genre spécifique que nous avons appréhendé à travers deux types de publications : d'une part les ouvrages du type "guides de la communication scientifique orale" publiés majoritairement en Grande-Bretagne et aux États-Unis par des scientifiques, à l'intention des autres chercheurs anglophones (Anholt, 1994; Davis, 1997; Fletcher, 1998; Hager \& Scheiber, 1997; Kenny, 1982; Vassallo, 1990); d'autre part les travaux de Rowley-Jolivet, dont la thèse et plusieurs articles publiés ultérieurement ont permis une meilleure connaissance de ce genre encore peu exploré. En outre, nous avons également procédé à des enregistrements vidéo de conférences scientifiques ;

Research into the patterns of communication in the general academic community as well as in the specific discourse communities [...] should definitely be seen as the concern of the ESP teacher. Furthermore, the process by which the findings of that research are applied to the preparation of teaching material and classroom teaching should be seen as the challenge and the stimulation of ESP teaching. (Dudley-Evans, 1993: 7)

\section{Expérimentation}

La première partie du projet a été consacrée à la recherche et à l'évaluation de l'existant en matière d'expression orale à but spécifique. Lors de cette période (ainsi que pendant la construction du cadre théorique), des ouvrages théoriques et pratiques, relevant de différents domaines, ont été consultés. Ces consultations par les membres de l'équipe ont donné lieu à des évaluations qui ont été enregistrées dans une base de données ${ }^{3}$, dont l'objectif était triple : le stockage des évaluations d'ouvrages ; l'information apportée aux enseignants ; l'information apportée aux étudiants.

Une analyse mixte des besoins a été conduite - par une enquête auprès des responsables de laboratoires et des directeurs d'école doctorale, et par des questionnaires auprès des 
utilisateurs potentiels (67 ayant été analysés en 1999-2000). Cette analyse a révélé la très forte motivation a priori des étudiants pour la formation proposée. Elle nous a permis de mettre en place une première expérimentation auprès d'étudiants de deuxième et troisième année de thèse de l'école doctorale Iaem (informatique, automatique, électronique et mathématiques).

Un système pédagogique alliant stages intensifs et travail en semi autonomie ${ }^{4}$ a été élaboré (voir la figure 1). La construction de ce système, dans lequel nous proposions un module en présentiel composé de deux stages intensifs distants de quelques mois, reposait sur l'hypothèse que la motivation forte exprimée par les apprenants devait leur permettre une prise de conscience de la nécessité de retravailler de façon individuelle en fonction des problèmes détectés au cours du premier stage ${ }^{5}$. Il nous semblait ainsi possible de les responsabiliser afin qu'ils prennent une partie de leur apprentissage en charge à l'issue du premier stage intensif.

Figure 1 - le système pédagogique mis en place à l'UHP

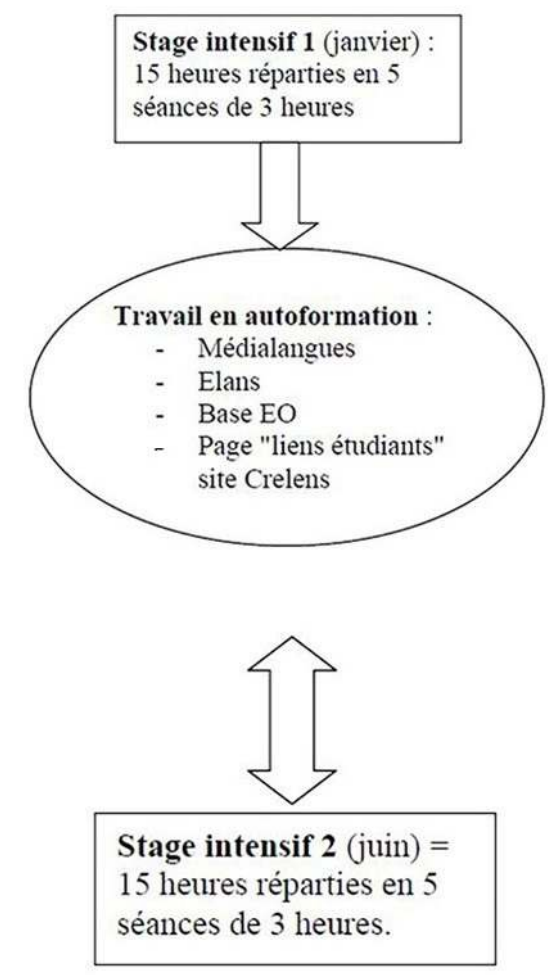

\subsection{Un premier module pilote}

Parce que les étudiants auraient à simuler une conférence orale, il y avait nécessité de constituer pour les stages des groupes réunissant les étudiants par spécialités scientifiques. En effet, afin de rapprocher le plus possible cette tâche d'une situation réelle, cette présentation devait être suivie d'un temps de questions (les autres membres du groupe pouvant poser des questions pertinentes à l'orateur). Or, il paraissait difficile de demander à un mathématicien, par exemple, de poser des questions pertinentes sur le travail de recherche d'un électronicien. En outre, la notation symbolique des différentes spécialités - tant sur le plan visuel, que linguistique - n'est pas la même. Le contenu 
pédagogique des stages intensifs comportait donc lui aussi des éléments spécifiques (travail sur la prononciation de signes spécifiques à telle ou telle spécialité, par exemple).

Les langues spécialisées scientifiques et techniques emploient non seulement une terminologie particulière mais également des graphies, ou signes non-linguistiques, spécifiques (notations mathématiques ou chimiques), et des éléments numériques et alphabétiques (alphabets romain et grec) [...] A l'oral la situation est plus complexe, car, si ces langages sont internationaux et translinguistiques à l'écrit $(+$ et - sont identiques en russe, anglais, français...), l'oralisation oblige à utiliser une langue donnée, l'anglais en l'occurrence (ainsi on dira 'minus' et non 'moins'...) et constitue donc une difficulté supplémentaire pour les locuteurs et auditeurs nonanglophones. (Rowley-Jolivet, 1997 : 233)

Les documents utilisés en cours ainsi que les activités impliquaient donc de tenir compte de la spécialité scientifique des étudiants concernés.

Des questionnaires bilans (utilisés à la fin du premier stage et à la fin du module) ont permis un retour sur le dispositif proposé. Ces retours montraient que les étudiants étaient globalement satisfaits sur le plan du contenu pédagogique (Chateau, 2003: 225-251). Néanmoins, la perte d'une partie des étudiants entre les deux stages (environ la moitié), ainsi que le fait que la moitié des étudiants ayant suivi tout le module avouaient ne pas avoir réellement retravaillé entre les deux stages (en dépit des incitations que nous leur avions prodiguées et des ressources dont ils disposaient pour le faire), nous ont amenés à nous interroger sur le phénomène de motivation et sur le fait qu'une mesure de la motivation préalable était probablement insuffisante (Chateau, 2005). En étudiant les réponses des étudiants au questionnaire distribué en fin de deuxième stage, ainsi que les messages envoyés par les étudiants absents à ce deuxième stage pour expliquer leur absence, nous avons constaté que de très fortes contraintes pesaient sur les doctorants et venaient en fait contrecarrer leur motivation ${ }^{6}$. Ceci nous a amenés à un questionnement sur la conception du dispositif proposé et à la mise en place d'une nouvelle expérimentation.

\subsection{Une formule modifiée}

Le dispositif mis en place la seconde année comportait un élément nouveau : 


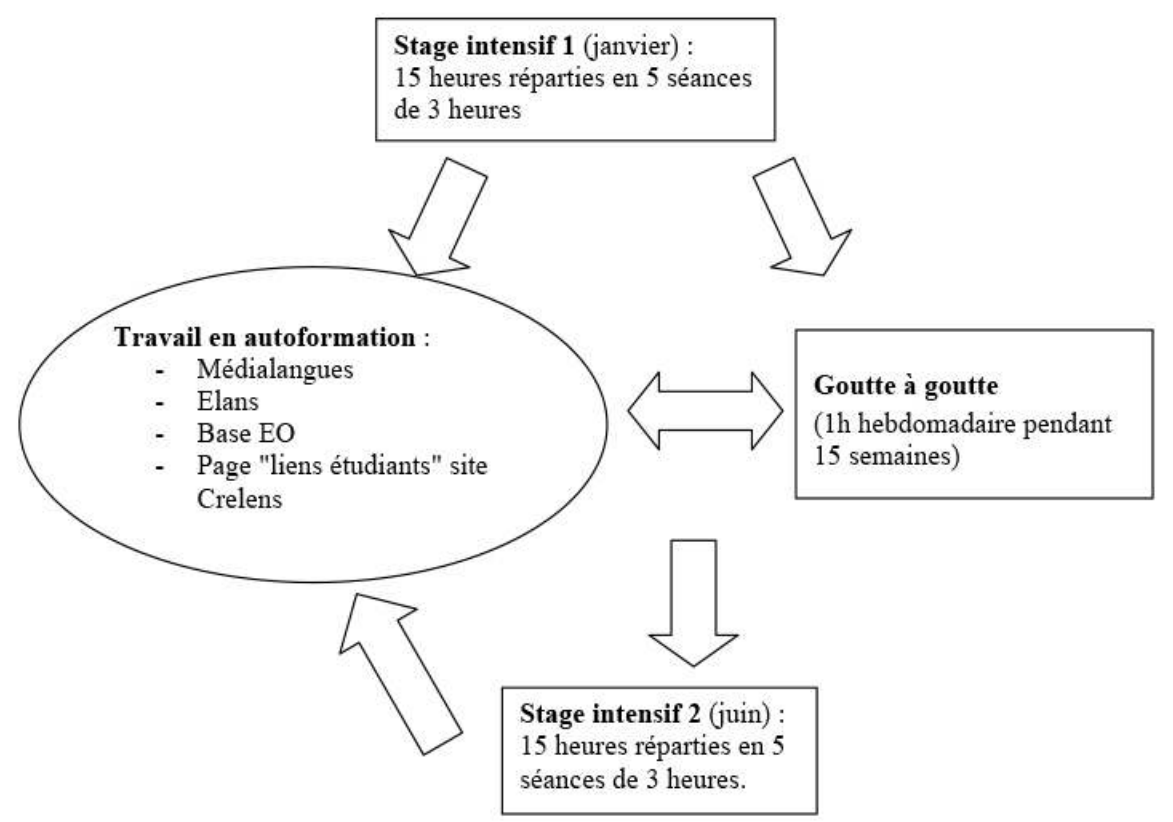

Comme l'indique la figure 2, l'équipe avait décidé de mettre en place un suivi d'environ une heure par semaine (appelé "goutte à goutte"7) entre les deux stages intensifs pour pallier les manques constatés lors de la première année d'expérimentation.

Les résultats obtenus au cours de l'année 2001 à l'aide de questionnaires du même type que ceux utilisés l'année précédente ont confirmé que les étudiants sondés étaient, comme ceux de l'année précédente, globalement satisfaits sur le plan du contenu pédagogique (à la fois du point de vue des informations apportées et des activités proposées). En outre, du point de vue de l'ensemble du dispositif, la seconde expérimentation a permis la validation de la formule modifiée. Il était donc possible de permettre au dispositif d'essaimer et de proposer le système conçu à une deuxième école doctorale.

\section{5. Élargissement}

En septembre 2000 le Crelens avait été sollicité par le directeur de l'école doctorale Sesames (Synthèses, Expériences, Simulations, Applications : de la Molécule aux Édifices Supramoléculaires), nouvellement créée, pour mettre en place un programme de formation, permettant, en deux années, aux étudiants inscrits en thèse dans les domaines scientifiques relevant de la chimie, de se former à la communication scientifique en anglais, à la fois écrite et orale.

\subsection{Une nécessaire adaptation}

D'une part, il s'agissait donc d'étudiants relevant d'un domaine scientifique différent de celui des étudiants auxquels nous nous étions déjà adressés. D’autre part, cette école doctorale, bien que dirigée par un professeur de l'UHP et regroupant des doctorants de cette université, est rattachée administrativement à l'université de Metz, et offre sa 
formation également à des doctorants messins. Grâce à l'expérience de recherche-action menée en Iaem, après discussion et analyse du problème, nous avons donc proposé au directeur de Sesames pour les étudiants inscrit en $2^{\text {ème }}$ année de thèse un dispositif similaire à celui mis en place lors des expérimentations menées les deux années précédentes. Pour les étudiants de $1^{\text {ère }}$ année un module spécifique a été conçu. Ce module est d'une durée de 30 heures (sous forme de deux stages intensifs de 15 heures chacun) et est axé essentiellement sur la rédaction scientifique ${ }^{8}$, la conception et la préparation de posters et l'expression orale à but général.

Une des différences majeures avec le dispositif mis en place grâce à la recherche-action menée en Iaem, outre les différences concernant les spécialités des étudiants et, par là même, la mise au point et l'adaptation nécessaire de certains des documents pédagogiques, est le fait que, dès le départ, le directeur de Sesames avait insisté pour que les deux modules soient obligatoires pour tous les étudiants de son école doctorale. $\mathrm{Ce}$ point était en contradiction avec certaines de nos hypothèses didactiques (parce qu'imposant une contrainte aux étudiants), notamment par rapport au rôle de l'émotion dans l'apprentissage (Chateau, 2003: 126-138). Les résultats obtenus, pourtant, ont illustré son impact positif, car il permettant d'alléger certaines contraintes professionnelles venant peser sur les étudiants (cf. section 4.1 ci-dessus).

Enfin, sur le plan pédagogique, le fait de savoir que les étudiants de cette école allaient être amenés à suivre un module s'étalant sur deux années universitaires et correspondant à un volume horaire de 75 heures en présenciel (deux stages de 15 heures en $1^{1 \text { ère }}$ année, deux stages de 15 heures en $2^{\text {ème }}$ année, plus 15 heures de 'goutte à goutte') nous a permis de mettre en place des stratégies et exercices dont l'efficacité nécessitent un travail de plus longue haleine que ce qu'il nous était possible de faire en Iaem.

\subsection{Permanence de certains éléments}

Bien qu'ayant adapté une partie de nos documents à la spécialité des étudiants, nous avons pu emporter dans d'autres formations une partie de l'information apportée aux étudiants au cours des stages intensifs. Ainsi nous avons utilisé, avec les étudiants de Sesames, les documents créés pour les étudiants d' Iaem présentant une série d'éléments autres que linguistiques. Ces documents, relevant avant tout du canal visuel (travail sur la gestuelle, l'utilisation des outils de communication) sont destinés à aider ces jeunes chercheurs à comprendre l'importance de ces paramètres non verbaux, qui peuvent faire la différence entre une communication réussie, et une communication qui ne remplit pas tout à fait ses objectifs, faute d'avoir su atteindre son public.

La structure du dispositif proposé à cette nouvelle école doctorale était globalement la même. Toutefois certains des doctorants (un peu moins de la moitié) effectuent leur thèse dans des laboratoires de l'université de Metz; cela nous a donc incité à "délocaliser" pour eux le système de guidage mis en place. Grâce à un collègue messin, précédemment en poste à l'UHP et membre de l'équipe originelle, nous avons pu néanmoins proposer à la totalité des étudiants de Sesames un dispositif quasiment similaire sur les deux sites.

\section{3. Évolution du système}

En 2002-2003, sous l'impulsion du directeur de Sesames qui avait souhaité dès le départ que les modules proposés aux étudiants de son école soient évalués au cours d'une 
"journée scientifique de rentrée" étudiants soient encore mieux préparés aux communications orales, le dispositif a été renforcé par une innovation que nous avons d'ailleurs introduite dans les deux écoles doctorales. Comme l'indique la figure 3, la première présentation orale - activité pivot, parce qu'elle sert de base au travail de remédiation individualisé mis en place dans la suite du dispositif et permet de montrer concrètement aux étudiants l'avantage qu'ils ont à se servir des outils dont nous leur avons recommandé l'utilisation (les outils permettant d'utiliser le code visuel pour pallier leurs éventuelles défaillances linguistiques) - a été repoussée de quelques semaines pour que les étudiants aient davantage de temps pour s'y préparer en fonction des conseils et suggestions qui leur sont donnés lors du premier stage.

Pour cette journée de simulation, nous faisons appel, pour chacune des deux écoles, à des collègues scientifiques. Ce travail en binôme a une double utilité, puisqu'il nous a permis à la fois de renforcer la spécificité de ces simulations selon les spécialités des étudiants, et - même si cela peut paraître paradoxal - de constater que les critères d'évaluation que nous utilisions pour juger de la qualité et de la clarté d'une communication étaient les mêmes que ceux des scientifiques (qu'il s'agisse d'un chimiste, directeur d'un laboratoire de recherche de l'UHP et d'une UMR, ou d'une collègue informaticienne, d'origine américaine).

Figure 3 - évolution du dispositif

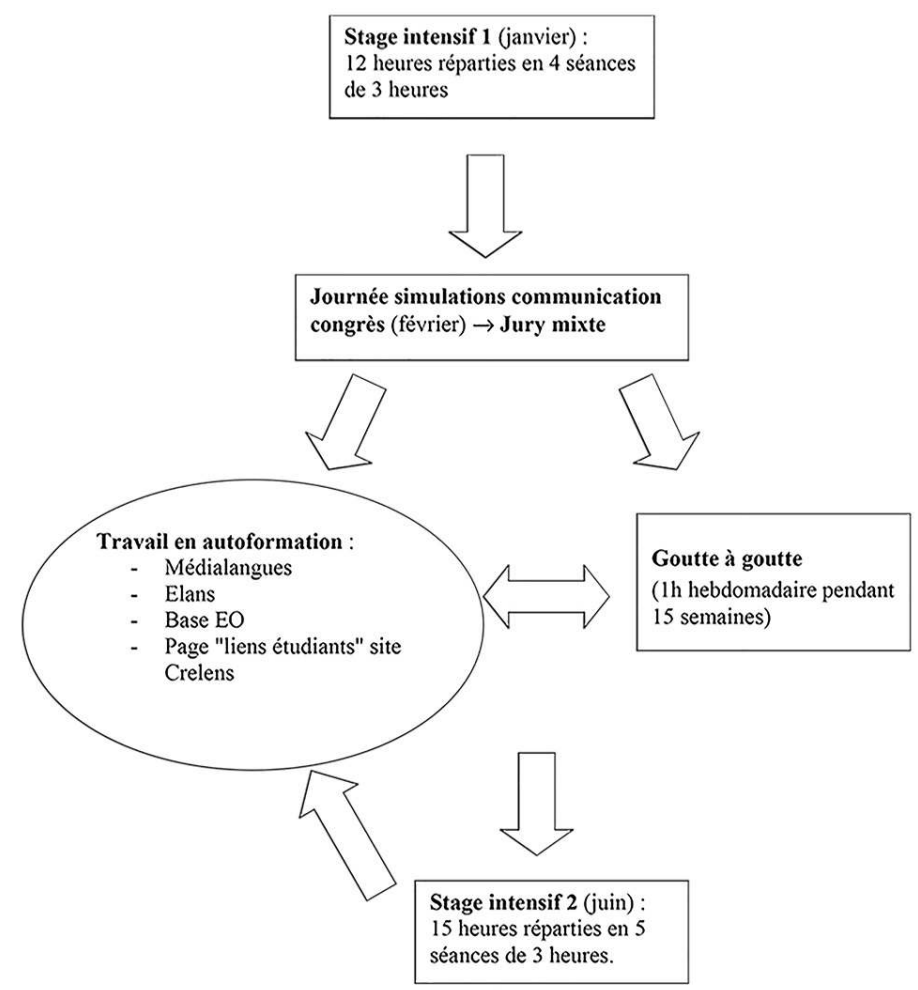

L'introduction de cette innovation - qui a permis la collaboration encore accrue des anglicistes avec leurs collègues scientifiques et le renforcement de la professionnalisation de la formation proposée aux étudiants - a donc, dans le contexte de l'UHP, mis en lumière le fait qu'une formation conçue pour un public spécifique et convenant aux besoins de ce public peut néanmoins comporter des paramètres généraux permettant son 
utilisation par un autre public. En outre, tout comme dans l'exemple rapporté par Péchou (2005 : 109), la crédibilité de l'enseignement des langues s'en est trouvée renforcée tant auprès des étudiants, qu'auprès de l'institution.

\section{Résultats et discussion}

La mise en place du dispositif dans une deuxième école doctorale peut être vue comme une extension ou un essaimage de la recherche-action menée, même si "une RechercheAction est une réponse à une situation particulière, [et que] le contexte dans lequel elle surgit reste singulier." (Catroux, 2002 : 15). L'adaptation aux besoins en fonction du contexte, ainsi que le nouveau type de validation obtenu dans le cas décrit ici, indiquent que le système créé est globalement transférable. On peut également signaler qu'une entreprise de formation en langues, ayant obtenu un contrat auprès d'un organisme national rassemblant des chercheurs et des professionnels d'une discipline médicale (la cancérologie), nous a consulté au moment de la conception des modules qu'elle souhaitait proposer à cet organisme, et que la conceptrice du dispositif proposé à cet organisme est même venue assister en tant qu'observatrice à certains des stages intensifs que nous avons proposés aux doctorants de l'UHP, ce qui est un autre indicateur de l'exportabilité d'une partie du système que nous avons mis sur pied.

En outre, du point de vue de l'apport au savoir, cette recherche a permis de constater qu'au niveau de la pratique, une mesure préalable de la motivation des apprenants est insuffisante car peu fiable et ne permettant pas de prévoir les contraintes du macro système susceptibles de venir la contrecarrer. Un nouveau type de mesure de la motivation tenant compte du paramètre temps semble donc nécessaire (Chateau, 2005).

Enfin, même s'il s'agit là plus de résultats obtenus dans le cadre de la pratique que lors d'un réel prolongement de la recherche précédemment décrite, l'utilisation de techniques et de documents pédagogiques créés pour les doctorants de l'UHP dans le cadre de deux modules mis en place à l'université Nancy 2, semble confirmer que le transfert du système est possible, si les deux publics ont des besoins communs. Le premier de ces modules a été mis en place dans le cadre du Dulasp (Diplôme universitaire de langue anglaise en situation professionnelle) créé par l'Université Nancy 2 en 2002 ; il s'adresse à des apprenants ayant un niveau minimum en anglais équivalent à celui d'un étudiant à $\mathrm{bac}+2$, souhaitant améliorer leurs compétences de communication professionnelle orale en anglais. Les origines professionnelles des apprenants inscrits à ce module pouvant être très variées, les éléments transférés concernaient donc essentiellement les techniques de communication orale. En 2004-2005 - dans le cadre du module d'anglais inscrit dans la formation de deuxième année des étudiants de Miage (Méthodes informatiques appliquées à la gestion des entreprises) de l'université Nancy 2 - j'ai été sollicitée pour préparer les étudiants à la présentation orale en anglais de leur projet IHM (Interface homme-machine). Cette préparation - dont l'objectif final est d'amener ces étudiants de niveau bac +3 à être capables d'exprimer en anglais leurs choix méthodologiques, décisions et conclusions - se fait en concertation avec leur enseignant d'IHM. Elle fut l'occasion de confirmer la validation des résultats de la recherche-action menée à l'UHP, puisqu'à l'issue des présentations effectuées par les étudiants, nous avons constaté que nos critères d'évaluation de la qualité et de la clarté des exposés étaient globalement les mêmes. 


\section{Conclusion}

La recherche menée a permis la mise en place d'une solution adaptée aux besoins : alors que rien n'était proposé pour répondre aux attentes des jeunes chercheurs en matière de communication orale en anglais à l'UHP, un système de guidage répondant à leurs besoins a été mis en place. Des outils adaptables aux étudiants de différentes spécialités et réutilisables ont également été créés, grâce à l'observation des habitudes de communication de la communauté scientifique en situation et à l'apport des nouvelles technologies. L'essaimage, réalisé tout d'abord à l'UHP, puis auprès d'autres publics, a montré l'adaptation possible des résultats de cette recherche à d'autres institutions ayant des besoins similaires.

Il semble donc que - même s'il ne fait aucun doute que la spécificité du vocabulaire et des contextes propres à chaque public est une réalité et doit être respectée - l'on peut en revanche tenter d'adapter dispositif et techniques pédagogiques créés pour un public précis à d'autres publics présentant des caractéristiques communes. L'apport de la recherche décrite ici est donc d'ordre méthodologique et social, et semble rejoindre les propos de Develay, pour qui "la recherche vise l'utilité sociale de ses produits" (2001 : 110).

\section{BIBLIOGRAPHIE}

Anholt, R. R. H. (1994). Dazzle 'em with style. The art of oral scientific presentation. New-York : W. H Freeman \& Company.

Catroux, M. (2002). "Introduction à la recherche-Action : modalités d'une démarche théorique centrée sur la pratique". Les Cahiers de l'APLIUT, vol. XXI, n³, pp. 8-20.

Chateau, A. (2003). Anglais de spécialité, la communication orale à objectifs spécifiques, une approche expérimentale du guidage. Thèse de Doctorat de l'Université de La Rochelle.

Consulté en janvier 2006 : http://halshs.ccsd.cnrs.fr/halshs-00005972.

Chateau, A. (2005). "La motivation, une question de mesure ?" Les Cahiers de l'APLIUT, vol. XXIV, n - 2, pp. 37-52.

Chateau, A. \& Georges, L. (2002). "La recherche-action : un moteur d'innovations pédagogiques? Analyse d'un cas concret". Les Cahiers de l'APLIUT, vol. XXI, n 3, pp. 66-80.

Davis, M. (1997). Scientific papers and presentations. San Diego: Academic Press.

Develay, M. (2001). Propos sur les sciences de l'éducation. Réflexions épistémologiques. Paris : ESF éditeur.

Dudley-Evans, T. (1993). "Subject Specificity in ESP: How much Does the Teacher Need to Know of the Subject?" ASp vol. 1, pp. 1-9.

Galisson, R. et Coste, D. (1976). Dictionnaire de didactique des langues. Paris : Hachette. 
Ginet, A. (1994). "Des transparents et des écrans qui communiquent ...en bleu Méditerranée". Asp, vol. 3, pp. 2-11.

Ginet, A. Kohlmayer, C., Narcy, J.-P., Northrup, L., \& Tassin, D. (1997). Du Laboratoire de langues à la salle de cours multimédias - de la recherche à la mise en pratique. Paris : Nathan.

Guyon, A. \& Guyon, E.(1996). "Anglais de spécialité et plurilinguisme". ASp, Vols. 11-14, pp. 1-11a.

Hager P. J. \& Scheiber H. J. (1997). Designing and Delivering Scientific, Technical, and Managerial Presentations. New York : John Wiley \& Sons.

Isani, S. (2004). "Compétence de culture professionnelle : définition, degrés et didactisation". Asp, Vols. 43/44, pp. 5-21.

Kenny, P. (1982). A Handbook of Public Speaking for Scientists and Engineers. Bristol : Institute of Physics Publishing.

Magnet, A. (1999). "Réseaux de cohérence et de cohésion dans la pratique de l'anglais scientifique". ASp, Vols. 23/26, pp. 85-98.

Narcy, J.-P. (1998). "La problématique 'Action Research' / Recherche Action et le travail coopératif". ASp, Vols. 19/22, pp. 229-238.

Narcy-Combes, J.-P. (2005). Didactique des Langues et TIC : vers une recherche-action responsable. Paris : Éditions Ophrys.

Narcy-Combes, M.-F. (2005). Précis de didactique. Devenir professeur de langue. Paris : Ellipses.

Ogrizek, M., Guillery, J.-M., Mirabaud, C.(1996). La communication médicale. Paris : Presses

Universitaires de France.

Péchou, A. (2005). "Aider à comprendre une conférence. Le cas du droit des contrats pour étayer la motivation". Les Cahiers de l'APLIUT, vol. XXIV, n 2, pp. 99-111.

Rowley-Jolivet, E. (1997) La Communication Scientifique Orale, Étude des Caractéristiques Linguistiques et Discursives d'un 'Genre'. Thèse de Doctorat de l'Université de Bordeaux 2.

Swales, J. M. (1990). Genre Analysis, English in academic and research settings. Cambridge: Cambridge University Press.

Vassallo, V. (1990). Speaking With Confidence: a Guide for Public Speakers. Whitehall : Betterway Books.

\section{NOTES}

1. LANgues pour Spécialistes d'Autres Disciplines.

2. Dues notamment au fait que les enseignants engagés dans le projet avaient tous un statut du second degré et que leur engagement, dans un projet prévu pour durer trois années universitaires s'était fait sur la base du quasi-volontariat, ce qui explique que certains aient du abandonner en cours de route.

3. Pour avoir une liste précise des ressources analysées consulter la "base eo", accessible depuis http :www.crelens.uhpnancy.fr.

4. "Lorsque l'autonomie ne concerne que certains aspects ou certains moments de l'apprentissage on peut parler de semiautonomie" (Galisson \& Coste, $1976: 62$ ).

5. Pour plus de détails sur les différentes ressources proposées pour le faire, voir Chateau, 2003 : 173-177. Il faut préciser que les étudiants s'inscrivaient au module sur la base du volontariat 
6. Ainsi, par exemple, le fait que certains directeurs de thèse refusent à la dernière minute de libérer leurs étudiants pour qu'ils puissent assister au stage d'anglais, pour cause de "manipulations urgentes à terminer au laboratoire".

7. Le nom que nous avions choisi pour la nouveauté introduite dans le système de guidage pour le renforcer nous semblait, dans le contexte d'une université scientifique, particulièrement approprié pour désigner l'innovation que nous allions introduire, dans la mesure où il s'agissait bel et bien d'instaurer une sorte de "perfusion" pour maintenir la motivation des étudiants entre les deux stages, et de faire en sorte qu'ils utilisent l'ensemble des ressources proposées pour améliorer leurs compétences de communication orale scientifique en anglais.

8. Afin d'adapter le plus possible ce module aux besoins des doctorants, nous leur demandons chaque année de nous fournir chacun un article en anglais faisant partie de leur bibliographie, et travaillons avec eux sur plusieurs de ces articles étudiés en parallèle, pour les sensibiliser à la notion de genre et à la structure spécifique des articles de recherche (cf. Swales, 1990).

9. Lors de cette journée le module proposé aux étudiants de 1ère année est validé par la réalisation d'un poster et un entretien avec le jury (devant ce poster) et le module proposé aux étudiants de 2ème année par une présentation orale en anglais de 7 minutes, suivie de 3 minutes de discussion devant l'ensemble des autres étudiants de l'école doctorale et devant les directeurs de thèse.

\section{RÉSUMÉS}

Une recherche-action auprès de doctorants scientifiques a confirmé l'importance de l'adaptation des formations proposées aux spécificités des publics. La finalité de ce projet fut la mise en place d'un système de guidage permettant d'améliorer l'expression orale en langue anglaise à buts spécifiques des jeunes chercheurs, a révélé que cette adaptation est impérative si l'on souhaiter respecter la motivation des apprenants, et répondre de la façon la plus adéquate possible à leurs besoins. L'essaimage de cette recherche cependant, a également indiqué qu'il était possible d'en adapter les résultats à d'autres publics possédant des caractéristiques connexes, pourvu que l'on tienne compte du contexte et du terrain spécifiques à chaque public, ainsi que de leurs exigences pratiques.

An action research project, whose aim was to improve the oral expression of doctoral students, emphasized the importance of adapting teaching methods and resources to the specific needs of the learners. The implementation of a guidance system to help students continue working on their own, and the adoption of the system by other learners, highlighted the necessity of respecting learners' needs in order to preserve their motivation. The results of this research can be applied to other learning situations, provided the specific context and its practical demands are taken into account.

\section{INDEX}

Keywords : ESP, action-research, context

Mots-clés : Lansad, recherche-action, contexte 


\section{AUTEUR}

\section{ANNE CHATEAU}

Anne Chateau a été de 1998 à 2004 chargée de mission langues, responsable de la gestion du Crelens (Centre de Ressources pour l'Enseignement des Langues Étrangères aux Non-Spécialistes) à l'université Henri Poincaré à Nancy. Après un doctorat de didactique de l'anglais de spécialité sous la direction de Jean-Paul Narcy-Combes, elle est, depuis septembre 2004, MCF à l'Université Nancy 2 au Scelv (Service Commun d'Enseignement des Langues Vivantes chargé de l'enseignement aux spécialistes d'autres disciplines). Sa recherche porte essentiellement sur la didactique de l'anglais pour spécialistes d'autres disciplines.

Courriel : anne.chateau[at]univ-nancy2.fr 